\title{
L'ancylostomose canine à Dakar
}

\author{
par P. MORNET, J. ORUE et M. SANE
}

L'ancylostomose canine est une affection banale à Dakar, diagnostiquée chaque année sur 200 à 300 chiens.

On connait le rôle joué par les larves d'ancylostomes pénetrant par voie cutanée chez l'homme qui provoquent des lésions dermo-épidermiques prurigineuses caractéristiques.

En saison chaude et humide où les plages sont très fréquentées, le "larbish 》 (nom wolof de cette affection particulière, appelée Pseudo-myiase rampante sous-cutanée, Roubaud, 1914, - infection par Larva migrans, Montel, 1951 - Creeping eruption aux U.S.A.) est très répandu, chez les enfants surtout, et les chiens qui solillent le sable de leurs excréments sont certainement responsables d'une grande partie des infestations.

\section{PARASTTE}

Les recherches systématiques entreprises depuis trois ans ont permis de rnettre on ćvidence chez les chiens parasités deux espèces de parasites :

Ancylostoma caninum et Ancylostoma brasiliense (1)

Le dernier est nettement plus répandu que le premier. En 1952, du 5 août au 29 décembre, nous avons fait l'autopsie de 96 chiens (provenant en grande partie de la fourrière municipale) et dénombré 5.651 ancylostomes. Sur ce nombre, 5.259 ćtaient des $A$. brasiliense, et 392 des $A$. caninum, soit une proportion de $93 \%$ pour les premiers.

Mâles et femelles de ces deux parasites ont été également classés :
A. brasiliense
1. 941
3.318
A. caninum. 146 246

\section{SYMPTOMES}

L'ancylostomose chez les chiens, à Dakar, ne constitue pas toujours une affection bien définie.

La symptomatologie est souvent fruste. L'inappétence et l'anémie sont les signes les plus constants.

(1) La détermination nous a été confirmée par M. le Professeur Guilhon, de l'Ecole Vetérinaire d'Alfort, que nous remercions vivement pour sa très grande obligeance.
Les autres, plus ou moins irrégulièrement observés et sans spécifité, sont : épistaxis, eternuements (?), vomissements, diarrhée. Certaines formes de purpura hemorragique pourraient être rapportées à l'ancylostomose, mais la demonstration n'en a pas éte faite.

Nous admettons qu'en principe, tout animal en mauvais état, affaibli, à appétit capricieux, à muqueuses buccale et conjonctivale décolorées, est suspect d'infestation et l'examen microscopique des selles est effectué systématiquement.

L'ancylostomose est souvent surajoutée à une affection qu'elle aggrave : maladie de Carré, infections intestinales, etc.

\section{RÉCEPTIVITÉ}

La race du chien ne semble pas avoir d'influence; les sujets autochtones sont infestés au même titre

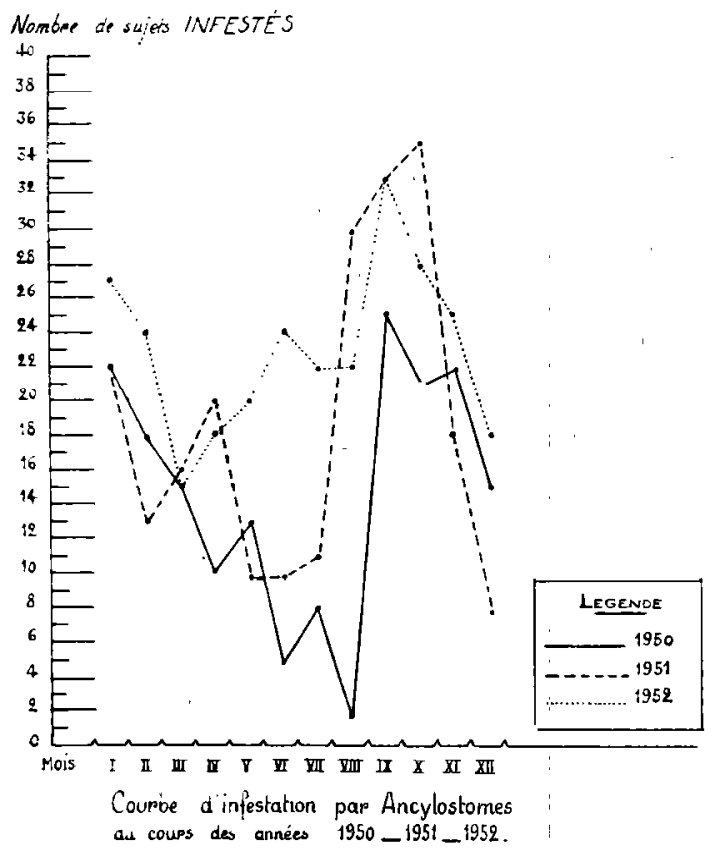

que ceux importés. Cependant, les chiens d'Europe vivant à Dakar supportent souvent mal la saison des 
pluies, de juillet à octobre, ce qui les rend plus sensibles à la maladie.

L'infestation est très variable suivant la saison : les mois pluvieux et humides, très favorables à l'éclosion des larves, sont ceux au cours desquels l'infestation se manifeste avec le plus d'intensité.

D'apres la courbe d'infestation établie pour les années 1950-1951-1952, les maxima sont notés pendant les mois de septembre et octobre (saison des pluies) et les minima en mars-décembre.

\section{DIAGNOSTIC}

Ainsi gue nous le notons plus haut, tout chien présenté d̀ la consultation pour "maigreur », inappétence, adynamie, est considéré, a priori, comme porteur d'ancylostomes. La recherche coprologique des œufs de parasites est facile et leur détermination aisée. Deux affections anémiantes doivent être différenciées de l'ancylostomose : la piroplasmose et la leishmaniose. Rien ne s'oppose d'ailleurs à ce que l'ancylostomose se superpose à ces pratozooses.

La facilité du diagnostic par l'examen coprologique nous fait négliger le test de formol-gélification (Malbrant, 1940) qui serait positif mais sans opalescence (contrairement à la leishmaniose).

\section{PRONOSTIC}

Il esi assez favorable si le diagnoslic est posé de façon précoce, avant que les signes de déchéance organique soient trop accusés, et le traitement approprié immédiatement appliqué. La ré-infestation est fréquente, aussi est-il indiqué de traiter systématiquement les chiens chaque année au moment de la principale période infestante.

Nous n'avons jamais constaté qu'une infestation sévère déterminait une immunité hautement protectrice (Otto G.-F., 1948).

\section{TRAITEMENT}

De nombreuses formules thérapeutiques sont préconisées. Elles sont excellentes au début de l'infestation ou si celle-ci est modérée. Les résultats sont moins bons si l'infestation est massive. Il est alors indiqué d'associer à l'anthelminthique une médication anti-anémique (martiale en particulier) et toni-cardiaque.
Nous avons expérimenté la valeur anthelminthique des produits suivants (1) :

- Tetrachloréthylène pur........ $0,2 \mathrm{~cm}^{3} / \mathrm{kg}$

- Thiodiphénylamine pure...... 25 à $50 \mathrm{cg} / \mathrm{kg}$ par jour pendant cinq jours.

- Diéthylcarbamyl - 1 méthyl - 4 pipérazine (2) : $5 \mathrm{cg} / \mathrm{kg}$ pendant deux jours consécutifs.

- Pipérazine (hydratée pure) en sirop à la dose de $5 \mathrm{cg} / \mathrm{kg}$ pendant trois jours consécutifs.

- Mandalate d'isoanyle en sirop : $1 \mathrm{~cm}^{3} / \mathrm{kg}^{\prime}$ pendant un à trois jours consécutifs.

Les résultats sont consignés dans les tableaux ci-contre.

Signalons, avant de les analyser, que les traitements expérimentaux n'ont pas été suivis de façon aussi rigoureuse que nous l'aurions souhaité, les exigences de la clinique quotidienne, absorbante, ot les autres travaux de laboratoire ne nous ayant pas permis de "suivre » les animaux pendant plusieurs semaines. La patience des propriétaires non plus...

Il s'en dégage cependant quelques éléments principaux :i

10 Le tétrachloréthylène semble le meilleur médicameni utilisé. Ce qui confirme l'expérience, déjà Jongue, que nous avons de ce produit.

$2^{\circ}$ Le mandalate d'isoamyle n'a pas été étudié : au moment de I'ingestion, et malgré l'excipient sirupeux, il produit des troubles «irritatifs » spectaculaires, et les propriétaires ne se montrent pas disposés à poursuivre le traitement. Nous supposons que les chiens seraient du même avis... s'ils pouvaient l'exprimer.

$3^{\circ}$ La pipérazine et le diéthylcarbamyl - I métbyl 4 pipérazine' - donnent des résultats superposables et médiocres.

$4^{0}$ La phénothiazine, malgré cinq jours consécutifs de traitement, n'entraine pas toujours la disparition des parasites dans les selles, ni mêne une anélioration clinique nette.

$5^{\circ}$ Le tétrachloréthylène, au contraire, sur 20 sujets traités pendant deux jours consécutifs seulement, conduit à la « stérilisation » coprologique dans 18 cas. Sur les 2 cas rebelles, un seul devrait être mis au passif de la médication ( $n^{\circ} 7$ ), la maladie de Carré, facteur « péjoratif », étant en fait responsable du deuxième.

Il est à remarquer la réaction de certains sujets (nos $1,2,4,5)$ à ce produit : troubles de l'équilibre, phénomènes, d'ailleurs passagers et sans conséquences, mais méritant d'être relevés.

(1) A la demande de M. le Professeur Guilhon.

(2) Notézine Specia. 


\section{CONCLUSION}

L'infestation des chiens par ancylostomes, fréquente à Dakar, en particulier pendant les mois chauds et humides, est due à deux parasites : Ancylostoma caninum et Ancylostoma brasiliense, le deuxième étant plus répandu que le premier.

La symptomatologie de l'ancylostomose, souvent fruste et parfois superposée à une affection intercurrente, se manifeste surtout par de l'inappétence, de l'adynamie, et de l'anémie. Des anthelminthiques utilisés expérimentalement (et dans la pratique ordinaire de la clinique) : tétrachloréthylène pur, phénothiazine, diéthylcarbamyl-méthyl-4 pipérazine (Notézine Spécia), pipérazine, c'est le tétrachloréthylène qui donne les meilleurs résulats.

Laboratoire fédéral de l'Élevage, à Dakar. 
I. - Traitement par IG

\begin{tabular}{|c|c|c|c|c|}
\hline RACE, SEXE ET AGE & $\begin{array}{l}\text { POIDS } \\
\text { en } \mathrm{kg}\end{array}$ & $\begin{array}{c}\text { DATE } \\
\text { de présentation }\end{array}$ & EXAMEN DES SELLES & SYMPTOMES \\
\hline $\begin{array}{l}\text { 1. - Chien berger alle- } \\
\text { mand, } 16 \text { mois. }\end{array}$ & 30 & $4-12-1951$ & $\begin{array}{c}\text { Ancylostomes } \\
+-+\end{array}$ & $\begin{array}{l}\text { Inappétence. Éternuements. Poil } \\
\text { terne. Congestion de la scléro- } \\
\text { tique. }\end{array}$ \\
\hline $\begin{array}{l}\text { 2. - Chien berger alle- } \\
\text { allemand, } 9 \text { mois. }\end{array}$ & 15 & $6-12-1951$ & $\begin{array}{c}\text { Ancylostomes } \\
+++\end{array}$ & $\begin{array}{l}\text { Hyperthermie. Inappétence. } \\
\text { Vomissements. Congestion de } \\
\text { la sclérotique. }\end{array}$ \\
\hline $\begin{array}{l}\text { 3. - Chien berger alle- } \\
\text { mand, } 18 \text { mois. }\end{array}$ & 33 & 15-12-1951 & $\begin{array}{c}\text { Ancylostomes } \\
+t\end{array}$ & $\begin{array}{l}\text { Amaigrissement. Inappétence. } \\
\text { Photophobie. Vomissements. } \\
\text { Diarrhée sanguinolente. }\end{array}$ \\
\hline $\begin{array}{l}\text { 4. - Chien berger alle- } \\
\text { mand, } 16 \text { mois. }\end{array}$ & 35 & $12-12-1951$ & $\begin{array}{c}\text { Mncylostomes } \\
+++\end{array}$ & $\begin{array}{l}\text { Inappétence, Congestion do la } \\
\text { sclérotique. }\end{array}$ \\
\hline $\begin{array}{l}\text { 5. - Chienne berger alle- } \\
\text { mand, I an., }\end{array}$ & 21 & 3-12-1951 & $\begin{array}{c}\text { Ancylostomes } \\
+\end{array}$ & $\begin{array}{l}\text { Congestion sclérotique. Inappé- } \\
\text { tence. Vomissements. Maladie } \\
\text { de Carré en Octobre } 1951 .\end{array}$ \\
\hline $\begin{array}{l}\text { 6. - Chien berger alle- } \\
\text { mand, } 18 \text { mois. }\end{array}$ & 20 & 3-12-1951 & $\begin{array}{c}\text { Ancylostomes } \\
\frac{1}{1}\end{array}$ & $\begin{array}{l}\text { Chute de poils et amaigrissement. } \\
\text { Inappétence. Vomissements. }\end{array}$ \\
\hline 7. - Chien ténériffe, 7 ans. & 11 & $24-12-1951$ & $\begin{array}{l}\text { Ancylostomes } \\
+ \\
\vdots\end{array}$ & $\begin{array}{l}\text { Présenté à la consultation pour } \\
\text { eczéma. Appétit capricieux. }\end{array}$ \\
\hline $\begin{array}{l}\text { 8. - Chienne samoyède, } \\
5 \text { ans. }\end{array}$ & 12 & 26-12-1951 & $\begin{array}{c}\text { Ancylostomes } \\
++\end{array}$ & $\begin{array}{l}\text { Présentée à la consultation pour } \\
\text { eczéma. Selles brun foncé. } \\
\text { Constipation. }\end{array}$ \\
\hline 9. - Chien griffon, 14 mois. & 10 & $26-10-1951$ & $\begin{array}{c}\text { Ancylostomes } \\
++\end{array}$ & $\begin{array}{l}\text { Amaigrissement. Éternuements. } \\
\text { Inappétence. Congestion de la } \\
\text { sclérotique. }\end{array}$ \\
\hline 10. - Chien fox croisé, 6 ans. & 12 & $22-11-1951$ & $\begin{array}{c}\text { Ancylostomes } \\
+++\end{array}$ & $\begin{array}{l}\text { Inappétence. Amaigrissement. } \\
\text { Anémie. Muqueuses blanc por- } \\
\text { celaine. Éternuements. }\end{array}$ \\
\hline
\end{tabular}




\section{strachloxéthylène pur}

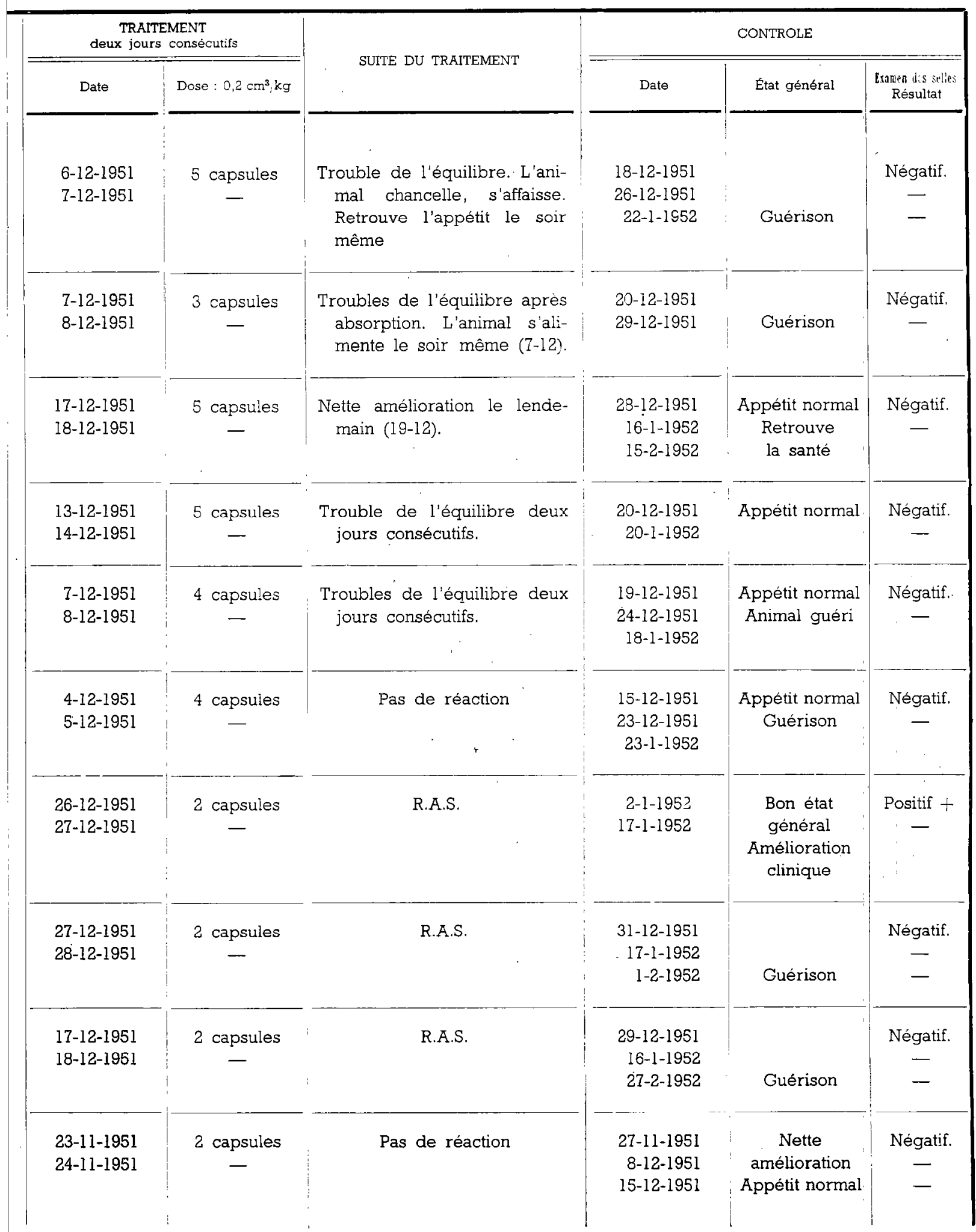


I. - Traitement

\begin{tabular}{|c|c|c|c|c|}
\hline RACE, SEXE ET AGE & $\begin{array}{l}\text { POIDS } \\
\text { en } \mathrm{kg}\end{array}$ & $\begin{array}{c}\text { DATE } \\
\text { de présentation }\end{array}$ & EXAMEN DES SELLES & SYMPTOMES \\
\hline $\begin{array}{l}\text { 11. - Chien de race indéter- } \\
\text { minée, poil long noir, } \\
6 \text { mois. }\end{array}$ & 13 & $5-12-1951$ & $\begin{array}{c}\text { Ancylostomes } \\
++\end{array}$ & Inappétence. \\
\hline 12. - Chien cocker, 1 an. & 12 & $6-12-1951$ & $\begin{array}{c}\text { Ancylostomes } \\
+\end{array}$ & $\begin{array}{l}\text { Maladie de Carré (forme pul- } \\
\text { monaire). }\end{array}$ \\
\hline $\begin{array}{l}\text { 13. - Chienne de race indé- } \\
\text { minee, } 8 \text { mois. }\end{array}$ & 9 & $18-12-1951$ & $\begin{array}{c}\text { Ancylostomes } \\
+\end{array}$ & Amaigrissement. Démodécie. \\
\hline 14. - Chien caniche, 13 mois. & 12 & $20-12-1951$ & $\begin{array}{c}\text { Ancylostomes } \\
+\end{array}$ & $\begin{array}{l}\text { Maladie de Carré. Hyperthermie. } \\
\text { Broncho-pneumonie. }\end{array}$ \\
\hline $\begin{array}{l}\text { 15. - Chien berger alle- } \\
\text { mand, } 11 \text { mois. }\end{array}$ & 17 & $24-12-1951$ & $\begin{array}{c}\text { Ancylostomes } \\
+\end{array}$ & $\begin{array}{l}\text { Inappétence. Amaigrissement. } \\
\text { Diarrhée sanguinolente. }\end{array}$ \\
\hline 16. - Chien fox, 2 ans. & 11 & $28-12-1051$ & $\begin{array}{l}\text { Ancylostomes } \\
++\end{array}$ & $\begin{array}{l}\text { Gale, sarcoptique. Anaigrisse- } \\
\text { ment. Inappétence. Congestion. } \\
\text { des muqueuses et de la scléro- } \\
\text { tique. }\end{array}$ \\
\hline $\begin{array}{l}\text { 17. - Chienne setter, } 2 \text { ans } \\
5 \text { mois. }\end{array}$ & 22 & $24-12-1951$ & $\begin{array}{c}\text { Ancylostomes } \\
+++\end{array}$ & $\begin{array}{c}\text { Vomissements et amaigrissement. } \\
\text { Congestion de la sclérotique. }\end{array}$ \\
\hline $\begin{array}{l}\text { 18. - Chienne bleu d'au- } \\
\text { vergne croisée, } 4 \text { ans. }\end{array}$ & 16 & $21-12-1951$ & $\begin{array}{c}\text { Ancylostomes } \\
+++\end{array}$ & $\begin{array}{l}\text { Anémie profonde. Muqueuses } \\
\text { blanc porcelaine. Amaigrisse- } \\
\text { ment considérable. Inappé- } \\
\text { tence. }\end{array}$ \\
\hline $\begin{array}{l}\text { 19. - Chien de race locale } \\
\text { froment clair, } 8 \text { mois. }\end{array}$ & 8 & $24-12-1951$ & $\begin{array}{c}\text { Ancylostomes } \\
+++\end{array}$ & $\begin{array}{l}\text { Amaigrissement. Inappetence. } \\
\text { Hyperthermie. }\end{array}$ \\
\hline $\begin{array}{l}\text { 20. - Chien épagneul, } \\
18 \text { mois. }\end{array}$ & 20 & $27-12-1951$ & $\begin{array}{c}\text { Ancylostomes } \\
+i\end{array}$ & Appétit capricieux. Tristesse. \\
\hline
\end{tabular}




\section{tétrachloréthylène pur (suite)}

\begin{tabular}{|c|c|c|c|c|c|}
\hline \multicolumn{2}{|c|}{$\begin{array}{c}\text { TRAITEMENT } \\
\text { deux jours consécutifs }\end{array}$} & \multirow{2}{*}{ SUITE DU TRAITEMENT } & \multicolumn{3}{|c|}{ CONTROLE } \\
\hline Date & Dose $: 0,2 \mathrm{~cm}^{3} \mathrm{~kg}$ & & Date & État général & $\begin{array}{l}\text { Exaraed des celles: } \\
\text { Résultat }\end{array}$ \\
\hline $\begin{array}{l}6-12-1951 \\
7-12-1951\end{array}$ & $\begin{array}{c}2 \text { capsules } \\
-\end{array}$ & Pas de réaction & $\begin{array}{l}15-12-1951 \\
24-12-1951\end{array}$ & $\begin{array}{c}\text { Nette } \\
\text { amélioration } \\
\text { Appétit normal }\end{array}$ & $\begin{array}{c}\text { Négatif. } \\
- \\
-\end{array}$ \\
\hline $\begin{array}{l}7-12-1951 \\
8-12-1951\end{array}$ & 2 capsules & Pas de réaction & $\begin{array}{l}15-12-1951 \\
22-12-1951\end{array}$ & Guérison & Négatif. \\
\hline $\begin{array}{l}19-12-1951 \\
20-12-1951\end{array}$ & 2 capsules & Pas de réaction & $\begin{array}{r}24-12-1951 \\
31-12-1951 \\
10-1-1952\end{array}$ & Guérison & $\begin{array}{c}\text { Négatif. } \\
- \\
-\end{array}$ \\
\hline $\begin{array}{l}21-12-1951 \\
22-12-1951\end{array}$ & $\begin{array}{c}2 \text { capsules } \\
-\end{array}$ & Pas de réaction & $\begin{array}{r}31-12-1951 \\
10-1-1952\end{array}$ & Guérison & $\begin{array}{c}\text { Négatif. } \\
-\end{array}$ \\
\hline $\begin{array}{l}26-12-1951 \\
27-12-1951\end{array}$ & $\begin{array}{c}3 \text { capsules } \\
-\end{array}$ & Pas de réaction & $\begin{array}{r}31-11-1951 \\
9-1-1952 \\
19-1-1952\end{array}$ & $\begin{array}{l}\text { Bon état } \\
\text { général } \\
\text { Guérison }\end{array}$ & $\begin{array}{c}\text { Négatif. } \\
- \\
-\end{array}$ \\
\hline $\begin{array}{l}29-12-1951 \\
30-12-1951\end{array}$ & $\begin{array}{c}2 \text { capsules } \\
-\end{array}$ & Pas de réaction & $\begin{array}{r}2-11-1951 \\
9-1-1952 \\
19-1-1952\end{array}$ & Guérison & $\begin{array}{l}\text { Négałif. } \\
\begin{array}{c}- \\
-\end{array}\end{array}$ \\
\hline $\begin{array}{c}26-12-1951 \\
28-12-1951 \\
\end{array}$ & $\begin{array}{c}4 \text { capsules } \\
-\end{array}$ & $\begin{array}{c}\text { Pas de réaction } \\
\text { Pas de vomissements }\end{array}$ & $\begin{array}{r}9-1-1952 \\
18-1-1952 \\
15-2-1952\end{array}$ & $\begin{array}{l}\text { Bon état } \\
\text { Guérison }\end{array}$ & $\begin{array}{l}\text { Négatif. } \\
\begin{array}{c}- \\
-\end{array}\end{array}$ \\
\hline $\begin{array}{l}26-12-1951 \\
27-12-1951\end{array}$ & $\begin{array}{c}3 \text { capsules } \\
-\end{array}$ & Pas de réaction & $\begin{array}{r}29-12-1951 \\
2-1-1952\end{array}$ & $\begin{array}{l}\text { Boulimie } \\
\text { Guérison }\end{array}$ & Négatif \\
\hline $\begin{array}{l}26-12-1951 \\
27-12-1951\end{array}$ & $\begin{array}{l}1 \text { capsule } \\
2 \text { capsules }\end{array}$ & Aucune réaction & $28-12-1951$ & $\begin{array}{c}\text { Pas } \\
\text { d'amélioration } \\
\text { la M. de Carré } \\
\text { ayant fait } \\
\text { son apparition }\end{array}$ & Positif + \\
\hline $\begin{array}{l}28-12-1951 \\
29-12-1951\end{array}$ & $\begin{array}{c}4 \text { capsules } \\
-\end{array}$ & Aucune réaction & $\begin{array}{r}9-1-1952 \\
31-1-1952 \\
27-2-1952\end{array}$ & $\begin{array}{l}\text { Appétit normal } \\
\text { Guérison }\end{array}$ & $\begin{array}{l}\text { Négatif. } \\
-\end{array}$ \\
\hline
\end{tabular}


II. - Traltement par la

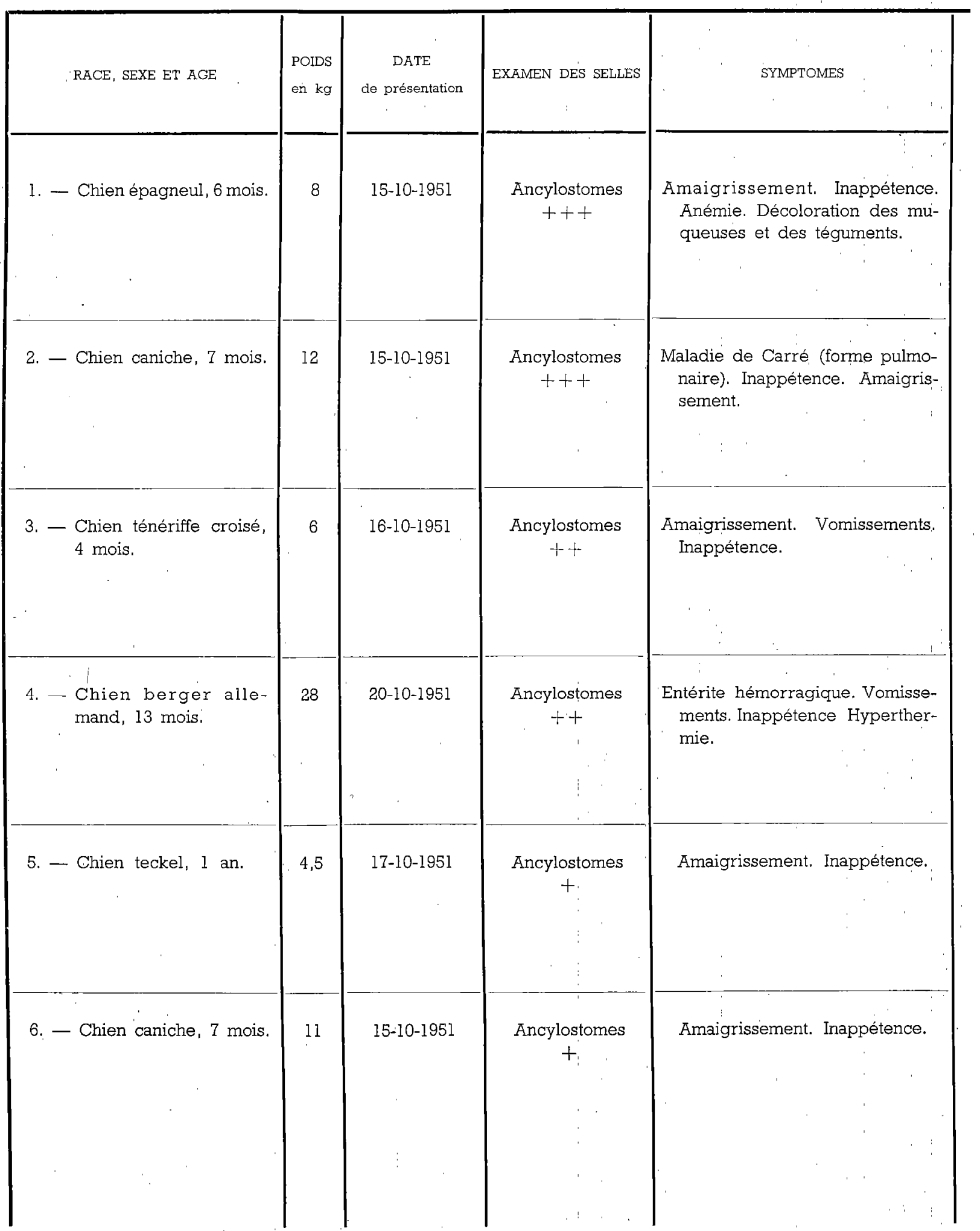




\section{phénothiazine (thiodiphénylamine)}

\begin{tabular}{|c|c|c|c|c|c|}
\hline \multicolumn{2}{|c|}{$\begin{array}{c}\text { TRAITEMENT } \\
\text { cinq jours consécutifs }\end{array}$} & \multirow[b]{2}{*}{ SUTTE DU TRAITEMENT } & \multicolumn{3}{|c|}{ CONTROLE } \\
\hline Date & Dose $: 0,25 \mathrm{~g} / \mathrm{kg}$ & & Date & Etat général & $\begin{array}{l}\text { Examerı } \\
\text { des selles } \\
\text { Résultat }\end{array}$ \\
\hline $\begin{array}{l}16-10-1951 \\
17-10-1951 \\
18-10-1951 \\
19-10-1951 \\
20-10-1951\end{array}$ & $\begin{array}{l}2 \mathrm{~g} \\
- \\
- \\
-\end{array}$ & Selles et urines rouges & $22-10-1951$ & $\begin{array}{l}\text { Amélioration } \\
\text { sensible } \\
\text { Appétit } \\
\text { retrouvé }\end{array}$ & Négatif. \\
\hline $\begin{array}{l}16-10-1951 \\
17-10-1951 \\
18-10-1951 \\
19-10-1951 \\
20-10-1951\end{array}$ & $\begin{array}{l}3 \mathrm{~g} \\
- \\
- \\
-\end{array}$ & Selles et urines rouges & $\begin{array}{c}22-10-1951 \\
25-10-1951 \\
2-11-1951 \\
24-12-1951 \\
29-1-1952\end{array}$ & $\begin{array}{c}\text { Grande } \\
\text { amélioration } \\
\text { Boulimie } \\
\text { Guérison }\end{array}$ & $\begin{array}{c}\text { Négatif. } \\
- \\
- \\
- \\
-\end{array}$ \\
\hline $\begin{array}{l}17-10-1951 \\
18-10-1951 \\
19-10-1951 \\
20-10-1951 \\
21-10-1951\end{array}$ & $\begin{array}{l}2 \mathrm{~g} \\
- \\
- \\
-\end{array}$ & Selles et urines rouges & $2-11-1951$ & Guérison & Négatif. \\
\hline $\begin{array}{l}21-10-1951 \\
22-10-1951 \\
23-10-1951 \\
24-10-1951 \\
25-10-1951\end{array}$ & $\begin{array}{l}8 \mathrm{~g} \\
- \\
- \\
-\end{array}$ & $\begin{array}{l}\text { Amélioration dès le lendemain } \\
\text { de l'administration. Rémis- } \\
\text { sion de tous les symptômes. } \\
\text { Selles et urines rouges. }\end{array}$ & $\begin{array}{r}28-10-1951 \\
2-11-1951 \\
11-11-1951 \\
17-11-1951\end{array}$ & $\begin{array}{l}\text { Guérison } \\
\text { clinique }\end{array}$ & $\begin{array}{l}\text { Négatif. } \\
\frac{-}{\overline{-}} \\
\text { Positif }\end{array}$ \\
\hline $\begin{array}{l}18-10-1951 \\
19-10-1951 \\
20-10-1951 \\
21-10-1951 \\
22-10-1951\end{array}$ & $\begin{array}{l}1 \mathrm{~g} \\
- \\
- \\
-\end{array}$ & Selles et urines rouges & $\begin{array}{l}31-10-1951 \\
22-11-1951\end{array}$ & $\begin{array}{l}\text { Appétit } \\
\text { et vigueur } \\
\text { Guérison }\end{array}$ & $\begin{array}{l}\text { Négatif. } \\
-\end{array}$ \\
\hline $\begin{array}{l}16-10-1951 \\
17-10-1951 \\
18-10-1951 \\
19-10-1951 \\
20-10-1951\end{array}$ & $\begin{array}{l}3 \mathrm{~g} \\
- \\
- \\
-\end{array}$ & $\begin{array}{l}\text { Crises épileptiformes. Selles } \\
\text { et urines rouges. Les crises } \\
\text { sont de plus en plus rappro- } \\
\text { chées, bien que l'animal soit } \\
\text { soumis au bromure. Les } \\
\text { crises épileptiformes n'ont } \\
\text { pas cessé et le chien a dis- } \\
\text { paru. }\end{array}$ & $20-10-1951$ & & Négatif. \\
\hline
\end{tabular}


II. - Traitement par la phénothiazine

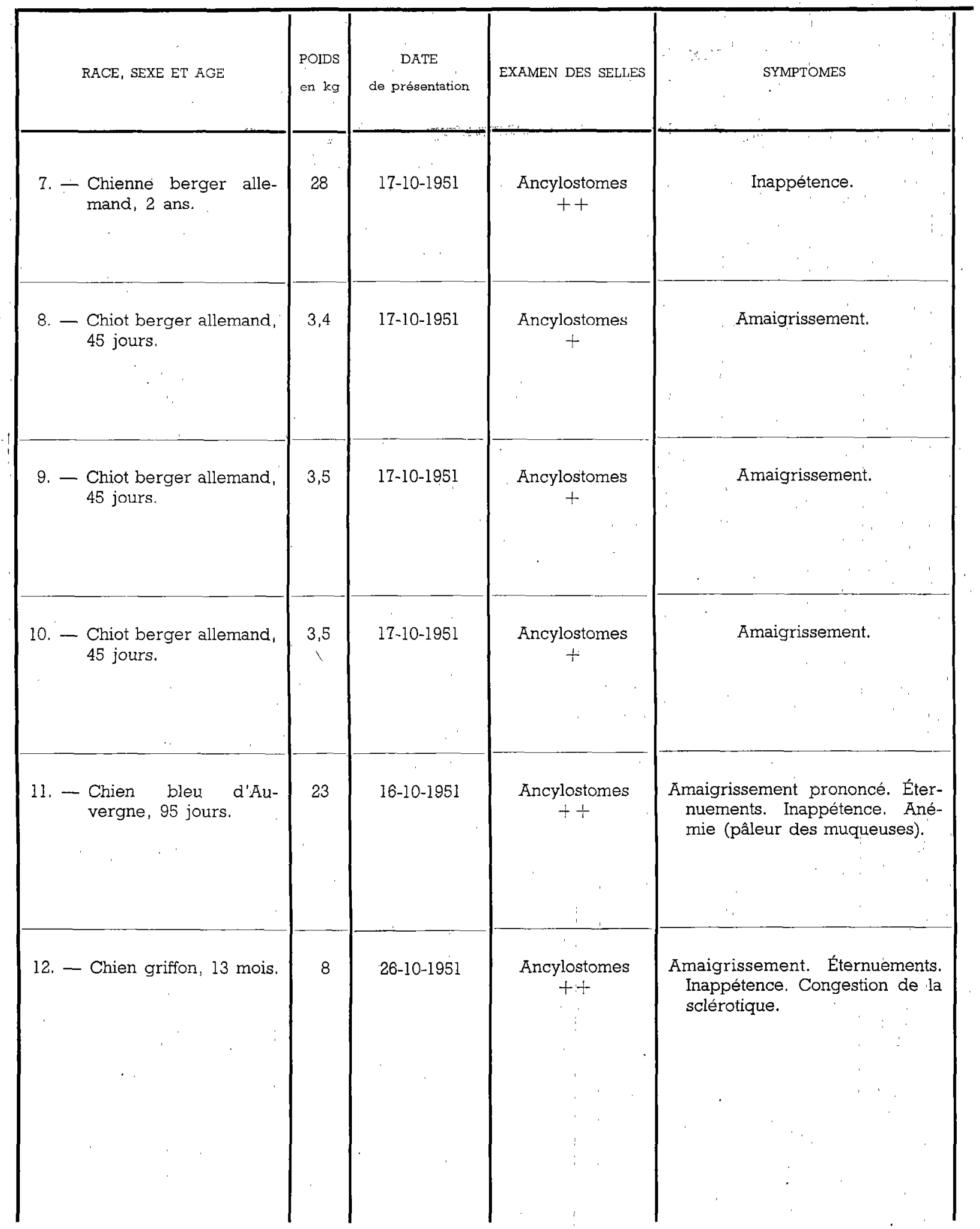


(thiodiphénglamine) (suite)

\begin{tabular}{|c|c|c|c|c|c|}
\hline \multicolumn{2}{|c|}{$\begin{array}{c}\text { TRAITEMENT } \\
\text { cinq jours consécutifs }\end{array}$} & \multirow{2}{*}{ "SUITE DU TRAITEMENT } & \multicolumn{3}{|c|}{ CONTROLE } \\
\hline Date & Dose : $0,25 \mathrm{~g} / \mathrm{kg}$ & & Date & État général & $\begin{array}{l}\text { Examon } \\
\text { des selles } \\
\text { Résultat }\end{array}$ \\
\hline $\begin{array}{l}17-10-1951 \\
18-10-1951 \\
19-10-1951 \\
21-10-1951\end{array}$ & $\begin{array}{l}8 \mathrm{~g} \\
-\end{array}$ & Selles et urines rouges & $\begin{array}{r}29-10-1951 \\
2-11-1951\end{array}$ & $\begin{array}{l}\text { Appétit normal } \\
\text { Guérison } \\
\text { clinique }\end{array}$ & Positif + \\
\hline $\begin{array}{l}17-10-1951 \\
18-10-1951 \\
19-10-1951 \\
20-10-1951 \\
21-10-1951\end{array}$ & $\begin{array}{l}19 \\
- \\
-\end{array}$ & Selles et urines rouges & $29-10-1951$ & $\begin{array}{l}\text { Léger } \\
\text { embonpoint } \\
\text { Guérison }\end{array}$ & Négatif. \\
\hline $\begin{array}{l}17-10-1951 \\
18-10-1951 \\
19-10-1951 \\
20-10-1951 \\
21-10-1951\end{array}$ & $\begin{array}{l}1 \mathrm{~g} \\
- \\
-\end{array}$ & Selles et urines rouges & $29-10-1951$ & $\begin{array}{l}\text { Bon état } \\
\text { Guérison }\end{array}$ & Négatif. \\
\hline $\begin{array}{l}17-10-1951 \\
18-10-1951 \\
19-10-1951 \\
20-10-1951 \\
21-10-1951\end{array}$ & $\begin{array}{l}19 \\
- \\
- \\
-\end{array}$ & Selles et urines rouges & $29-10-1951$ & $\begin{array}{l}\text { Bon état } \\
\text { Guérison }\end{array}$ & Négatif. \\
\hline $\begin{array}{l}17-10-1951 \\
18-10-1951 \\
19-10-1951 \\
20-10-1951 \\
21-10-1951\end{array}$ & $\begin{array}{l}4 \subseteq \\
- \\
- \\
-\end{array}$ & $\begin{array}{l}\text { Grande faiblesse le } 22-10 . \\
\text { Prostration. Anémie pronon- } \\
\text { cée. Muqueuses blanc por- } \\
\text { celaine. Température } 38^{\circ} \text {. } \\
\text { Selles et urines rouges. }\end{array}$ & $22-10-1951$ & $\begin{array}{l}\text { Animal mort } \\
\text { quelques } \\
\text { jours après. } \\
\text { L'autopsie } \\
\text { n'a pas été } \\
\text { pratiquée. }\end{array}$ & Négatif. \\
\hline $\begin{array}{l}27-10-1951 \\
28-10-1951 \\
29-10-1951 \\
30-10-19 E 1 \\
31-10-1951\end{array}$ & $\begin{array}{l}2 \mathrm{~g} \\
- \\
-\end{array}$ & $\begin{array}{l}\text { Paralysé le 2-11. Selles et } \\
\text { urines rouges. Polyurie } \\
\text { (troubles sphinctériens). }\end{array}$ & $\begin{array}{l}2-11-1951 \\
3-11-1951 \\
8-11-1951\end{array}$ & $\begin{array}{l}\text { Échec du trai- } \\
\text { tement coïn- } \\
\text { cidant avec } \\
\text { l'apparition } \\
\text { de la Mala- } \\
\text { die de Car- } \\
\text { ré, respon- } \\
\text { sable de la } \\
\text { paraplégie } \\
\text { et des trou- } \\
\text { bles sphinc- } \\
\text { tériens. }\end{array}$ & $\begin{array}{l}\text { Positif. } \\
- \\
-\end{array}$ \\
\hline
\end{tabular}


II. - Traitement par la phénothiazine

\begin{tabular}{|c|c|c|c|c|}
\hline RACE, SEXE ET.AGE & $\begin{array}{l}\text { POIDS } \\
\text { en } \mathrm{kg}\end{array}$ & $\begin{array}{c}\text { DATE } \\
\text { de présentation }\end{array}$ & EXAMEN DES SELLES & SYMPTOMES \\
\hline $\begin{array}{l}\text { 13. - Chien berger alle- } \\
\text { mand, } 10 \text { mois. }\end{array}$ & 20 & 5-1 1-1951 & $\begin{array}{c}\text { Ancylostomes } \\
+++\end{array}$ & $\begin{array}{l}\text { Hyperthermie. Amaigrissement. } \\
\text { Éternuements et léger jetage. } \\
\text { Toux discrète. Inappétence. } \\
\text { (Maladie de Carré). }\end{array}$ \\
\hline 14. - Chien ténériffe, 7 mois. & 4 & 18-10-1951 & $\begin{array}{c}\text { Ancylostomes } \\
+\end{array}$ & $\begin{array}{l}\text { Éternuements et vomissements. } \\
\text { : Entérite. Amaigrissement. }\end{array}$ \\
\hline $\begin{array}{l}\text { 15. - Chien de race indéter- } \\
\text { minée, noir, extrémités } \\
\text { feu, } 11 \text { mois. }\end{array}$ & 17 & 18-10-1951 & $\begin{array}{c}\text { Ancylostomes } \\
++\end{array}$ & Inappétence. \\
\hline 16. - Chien ténériffe, 7 mois. & 7 & 18-10-1951 & $\begin{array}{c}\text { Ancylostomes } \\
++\end{array}$ & $\begin{array}{l}\text { Amaigrissement et crises épilep- } \\
\text { tiformes. Maladie 'de Carré } \\
\text { (forme nerveuse). }\end{array}$ \\
\hline $\begin{array}{l}\text { 17. - Chienne épagneul, } \\
18 \text { mois. }\end{array}$ & 26 & $6-11-1951$ & $\begin{array}{c}\text { Ancylostomes } \\
++\end{array}$ & $\begin{array}{l}\text { Amaigrissement. Inappétence. } \\
\text { Vomissements. Congestion de } \\
\text { la sclérotique. }\end{array}$ \\
\hline $\begin{array}{l}\text { 18. - Chien berger alle- } \\
\text { mand, } 16 \text { mois. }\end{array}$ & 35 & 17-11-1951 & $\begin{array}{c}\text { Ancylostomes } \\
+++\end{array}$ & $\begin{array}{l}\text { Inappétence. Hyperthermie. Con- } \\
\text { gestion de la sclérotique. }\end{array}$ \\
\hline
\end{tabular}




\begin{tabular}{|c|c|c|c|c|c|}
\hline \multicolumn{2}{|c|}{$\begin{array}{c}\text { TRAITEMENT } \\
\text { cinq jours consécutifs }\end{array}$} & \multirow{2}{*}{ SUITE DU TRAITEMENT } & \multicolumn{3}{|c|}{ CONTROLE } \\
\hline Date & Dose : $0,25 \mathrm{~g}: \mathrm{kg}$ & & Date & État géneral & $\begin{array}{l}\text { Examen } \\
\text { des selles } \\
\text { Résultat }\end{array}$ \\
\hline $\begin{array}{r}6-11-1951 \\
7-11-1951 \\
8-11-1951 \\
9-11-1951 \\
10-11-1951\end{array}$ & $\begin{array}{l}5 \mathrm{~g} \\
- \\
- \\
-\end{array}$ & Selles et urines rouges & $\begin{array}{l}12-11-1951 \\
26-11-1951 \\
15-12-1951\end{array}$ & $\begin{array}{l}\text { L'animal man- } \\
\text { ge et joue. } \\
\text { Guérison de } \\
\text { la Maladie } \\
\text { de Carré et } \\
\text { de l'Ancy- } \\
\text { lostomose. }\end{array}$ & $\begin{array}{l}\text { Négatif. } \\
- \\
-\end{array}$ \\
\hline $\begin{array}{l}18-10-1951 \\
19-10-1951 \\
20-10-1951 \\
21-10-1951 \\
22-10-1951\end{array}$ & $\begin{array}{l}1 \mathrm{~g} \\
- \\
- \\
-\end{array}$ & Selles et urines rouges & $\begin{array}{l}24-10-1951 \\
13-11-1951\end{array}$ & $\begin{array}{l}\text { Bon état } \\
\text { Guérison } \\
\text { clinique }\end{array}$ & $\begin{array}{l}\text { Négatif. } \\
\text { Positif }+\end{array}$ \\
\hline $\begin{array}{l}18-10-1951 \\
19-10-1951 \\
20-10-1951 \\
21-10-1951 \\
22-10-1951\end{array}$ & $\begin{array}{l}4 \mathrm{~g} \\
- \\
- \\
-\end{array}$ & Selles et urines rouges & $3-11-1951$ & Bon appétit & Négatif. \\
\hline $\begin{array}{l}19-10-1951 \\
20-10-1951 \\
21-10-1951 \\
22-10-1951 \\
23-10-1951\end{array}$ & $\begin{array}{l}2 \mathrm{~g} \\
- \\
- \\
-\end{array}$ & Selles et urines rouges & $\begin{array}{l}31-10-1951 \\
14-11-1951\end{array}$ & $\begin{array}{l}\text { Légère } \\
\text { amélioration }\end{array}$ & $\begin{array}{l}\text { Négatif. } \\
\text { Positif }+\end{array}$ \\
\hline $\begin{array}{r}7-11-1951 \\
8-11-1951 \\
9-11-1951 \\
10-11-1951 \\
12-11-1951\end{array}$ & $\begin{array}{l}4 \mathrm{~g} \\
- \\
- \\
-\end{array}$ & $\begin{array}{l}\text { Amélioration nette } \\
\text { Selles et urines rouges }\end{array}$ & $\begin{array}{l}15-11-1951 \\
30-11-1951 \\
27-12-1951\end{array}$ & $\begin{array}{l}\text { Amendement } \\
\text { des symptô- } \\
\text { mes. Appé- } \\
\text { tit, ma is } \\
\text { pâleur dos } \\
\text { muqueuses. }\end{array}$ & $\begin{array}{l}\text { Négatif. } \\
\text { Positif }+ \\
-\end{array}$ \\
\hline $\begin{array}{l}18-11-1951 \\
19-11-1951 \\
20-11-1951 \\
21-11-1951 \\
22-11-1951\end{array}$ & $\begin{array}{l}8 \mathrm{~g} \\
- \\
- \\
-\end{array}$ & Selles et urines rouges & $30-11-1951$ & $\begin{array}{l}\text { Bon appétit } \\
\text { et vigueur } \\
\text { Guérison } \\
\text { clinique }\end{array}$ & Positif + \\
\hline
\end{tabular}


III. - Traitement par le diéthylcar-

\begin{tabular}{|c|c|c|c|c|}
\hline RACE, SEXE ET AGE & $\begin{array}{l}\text { POIDS } \\
\text { en } \mathrm{kg}\end{array}$ & $\begin{array}{l}\text { DATE } \\
\text { de présentation }\end{array}$ & EXAMEN DES SELLES & SYMPTOMES \\
\hline $\begin{array}{l}\text { 1. - Chien berger alle- } \\
\text { mand, } 6 \text { mois. }\end{array}$ & 14 & $7-9-1951$ & $\begin{array}{c}\text { Ancylostomes } \\
+++\end{array}$ & Bon appétit. Vomissements. \\
\hline $\begin{array}{l}\text { 2. Chien berger alle- } \\
\text { mand, } 15 \text { mois. }\end{array}$ & 33 & $7-9-1951$ & $\begin{array}{c}\text { Ancylostomes } \\
++\end{array}$ & Ancylostomose rebelle. \\
\hline $\begin{array}{l}\text { 3. - Chien caniche, } 5 \text { mois } \\
\text { et demi. }\end{array}$ & $.13,5$ & $8-9-1951$ & $\begin{array}{c}\text { Ancylostomes } \\
+\end{array}$ & Pas de signes cliniques. \\
\hline $\begin{array}{l}\text { 4. - Chien berger alle- } \\
\text { mand, } 2 \text { mois et demi. }\end{array}$ & 4 & $8-9-1951$ & $\begin{array}{c}\text { Ancylostomes } \\
++\end{array}$ & Amaigrissement. \\
\hline $\begin{array}{l}\text { 5. - Chien caniche marron, } \\
1 \text { an. }\end{array}$ & 13 & $10-9-1951$ & $\begin{array}{c}\text { Ancylostomes } \\
+\end{array}$ & Maladie de Carré. Kératite. \\
\hline 6. - Chien caniche, 6 mois. & 13 & $11-9-1951$ & $\begin{array}{c}\text { Ancylostomes } \\
++\end{array}$ & $\begin{array}{l}\text { Vomissements. Diarrhée glai- } \\
\text { reuse: }\end{array}$ \\
\hline $\begin{array}{l}\text { 7. - Chien dogue de Bor- } \\
\text { deaux, } 25 \text { mois. }\end{array}$ & 40 & $11-9-1951$ & $\begin{array}{c}\text { Ancylostomes } \\
+\end{array}$ & $\begin{array}{l}\text { Arraigrissement. Epistaxis. Ané- } \\
\text { mie. }\end{array}$ \\
\hline $\begin{array}{l}\text { 8. - Chien berger alle- } \\
\text { mand croisé, } 14 \text { mois. }\end{array}$ & 26 & $13-9-1951$ & $\begin{array}{c}\text { Ancylostomes } \\
++\end{array}$ & $\begin{array}{l}\text { Pas de signes cliniques. Vomis- } \\
\text { sements et congestion de la } \\
\text { sclérotique. }\end{array}$ \\
\hline $\begin{array}{l}\text { 9. - Chien berger alle- } \\
\text { mand, } 4 \text { mois et demi. }\end{array}$ & 17 & $13-9-1951$ & $\begin{array}{c}\text { Ancylostomes } \\
+\end{array}$ & $\begin{array}{l}\text { Vomissement. Diarrhée. Abatte- } \\
\text { ment. }\end{array}$ \\
\hline $\begin{array}{l}\text { 10. - Chien berger malinois, } \\
2 \text { ans et demi. }\end{array}$ & 26 & 14-9-1951 & $\begin{array}{c}\text { Ancylostomes } \\
++\end{array}$ & Anémie, Maigreur. Inappétence. \\
\hline $\begin{array}{l}\text { 11. - Chien berger alle- } \\
\text { mand, } 4 \text { mois et demi. }\end{array}$ & 17 & $15-9-1951$ & $\begin{array}{c}\text { Ancylostomes } \\
++\end{array}$ & Hyperthermie, \\
\hline $\begin{array}{l}\text { 12. - Chien berger alle- } \\
\text { mand, } 8 \text { mois. }\end{array}$ & 22 & $15-9-1951$ & $\begin{array}{c}\text { Ancylostomes } \\
+\end{array}$ & Vomissements. Inappétence. \\
\hline $\begin{array}{l}\text { 13. - Chien berger alle- } \\
\text { mand, } 18 \text { mois. }\end{array}$ & 21 & $21-9-1951$ & $\begin{array}{c}\text { Ancylostomes } \\
+\end{array}$ & Éternuements. Amaigrissement. \\
\hline 14. - Chien caniche, 3 mois. & 15 & $19-9-1951$ & $\begin{array}{c}\text { Ancylostomes } \\
+\end{array}$ & $\begin{array}{l}\text { Hyperthermie. Anorexie. Conges- } \\
\text { tion des muqueuses. }\end{array}$ \\
\hline
\end{tabular}


bamyl-1-méthyl-4-piperazine

\begin{tabular}{|c|c|c|c|c|c|}
\hline \multicolumn{2}{|c|}{$\begin{array}{c}\text { TRATTEMENT } \\
\text { deux jours consécutifs }\end{array}$} & \multirow{2}{*}{ SUITE DU TRAITEMENT } & \multicolumn{3}{|c|}{ CONTROLE } \\
\hline Date & $\begin{array}{l}\text { Dose }: 5 \mathrm{cg}_{i} \mathrm{~kg} \\
\text { capsule }=0.10\end{array}$ & & Date & État général & $\begin{array}{l}\text { Exampan des selle: } \\
\text { Résulftat }\end{array}$ \\
\hline $\begin{array}{l}7-9-1951 \\
8-9-1951\end{array}$ & $\begin{array}{c}7 \text { capsules } \\
-\end{array}$ & R.A.S. & $\begin{array}{l}10-9-1951 \\
13-9-1951\end{array}$ & Bon état & $\begin{array}{c}\text { Positif } \\
-\end{array}$ \\
\hline $\begin{array}{l}7-9-1951 \\
8-9-1951\end{array}$ & 16 capsules & R.A.S. & $11-9-1951$ & $\begin{array}{l}\text { Méme état } \\
\text { de maigreur }\end{array}$ & Positif + \\
\hline $\begin{array}{l}8-9-1951 \\
9-9-1951\end{array}$ & 6 capsules & R.A.S. & $15-9-1951$ & $\begin{array}{c}\text { Légère } \\
\text { amélioration }\end{array}$ & Positif + \\
\hline $\begin{array}{r}9-9-1951 \\
10-9-1951\end{array}$ & 2 capsules & R.A.S. & $13-9-1951$ & Mauvais état & Négatif. \\
\hline $\begin{array}{l}11-9-1951 \\
12-9-1951\end{array}$ & 6 capsules & R.A.S. & $12-9-1951$ & $\begin{array}{l}\text { Légère } \\
\text { amélioration }\end{array}$ & Positif. \\
\hline $\begin{array}{l}11-9-1951 \\
12-9-1951\end{array}$ & $\begin{array}{c}6 \text { capsules } \\
-\end{array}$ & R.A.S. & $\begin{array}{l}14-9-1951 \\
17-9-1951\end{array}$ & $\begin{array}{c}\text { Nette } \\
\text { amélioration }\end{array}$ & $\begin{array}{c}\text { Négatif. } \\
-\end{array}$ \\
\hline $\begin{array}{l}11-9-1951 \\
12-9-1951\end{array}$ & 20 capsules & $\begin{array}{l}\text { Aucune réaction. Epistaxis dans } \\
\text { la soirée. }\end{array}$ & $17-9-1951$ & $\begin{array}{c}\text { Même état } \\
\text { d'amaigrisse- } \\
\text { ment }\end{array}$ & Négatit. \\
\hline $\begin{array}{l}13-9-1951 \\
14-9-1951\end{array}$ & 13 capsules & Ptyalisme & $17-9-1951$ & $\begin{array}{c}\text { Amélioration } \\
\text { des symptômes }\end{array}$ & Positif: + \\
\hline $\begin{array}{l}13-9-1951 \\
14-9-1951\end{array}$ & $\begin{array}{l}8 \text { capsules } \\
-\end{array}$ & Ptyalisme & $\begin{array}{l}18-9-1951 \\
22-9-1951\end{array}$ & $\begin{array}{l}\text { Amélioration } \\
\text { notable } \\
\text { Vitalité }\end{array}$ & $\begin{array}{c}\text { Positif. } \\
-\end{array}$ \\
\hline $\begin{array}{l}14-9-1951 \\
15-9-1951\end{array}$ & 13 capsules & R.A.S. & $18-9-1951$ & $\begin{array}{l}\text { Nette amélio- } \\
\text { ration. Ani- } \\
\text { mal mange } \\
\text { à nouveau. }\end{array}$ & Négatif. \\
\hline $\begin{array}{l}15-9-1951 \\
16-9-1951\end{array}$ & 8 capsules & R.A.S. & $20-9-1951$ & $\begin{array}{l}\text { Légère } \\
\text { amélioration }\end{array}$ & Positif. \\
\hline $\begin{array}{l}16-9-1951 \\
17-9-1951\end{array}$ & 11 capsules & R.A.S. & $28-9-1951$ & $\begin{array}{c}\text { Amendement } \\
\text { des symptômes }\end{array}$ & Négatif. \\
\hline $\begin{array}{l}22-9-1951 \\
23-9-1951\end{array}$ & 10 capsules & Ptyalisme & $24-9-1951$ & $\begin{array}{c}\text { Légère } \\
\text { amélioration }\end{array}$ & Négatif. \\
\hline $\begin{array}{l}20-9-1951 \\
21-9-1951\end{array}$ & 8 capsules & & $2-10-1951$ & $\begin{array}{c}\text { Notable } \\
\text { amelioration }\end{array}$ & Négatif. \\
\hline
\end{tabular}




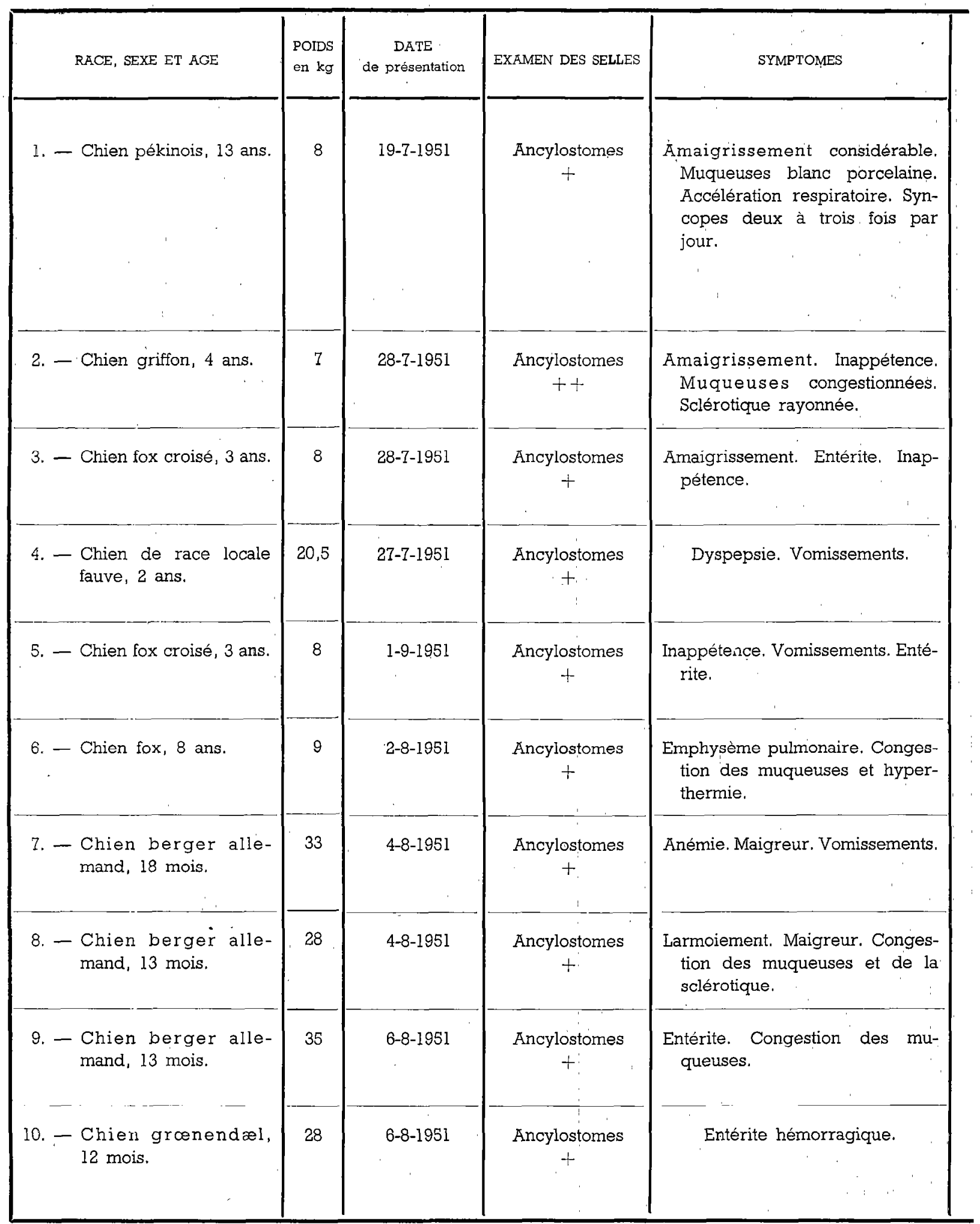


par la piperazine

\begin{tabular}{|c|c|c|c|c|c|}
\hline \multicolumn{2}{|c|}{$\begin{array}{l}\text { TRAITEMENT } \\
3 \text { jours consécutifs }\end{array}$} & \multirow{2}{*}{ SUITE DU TRAITEMENT } & \multicolumn{3}{|c|}{ CONTROLE } \\
\hline Date & Dose $5 \mathrm{cg}^{\prime} \mathrm{kg}$ & & Date & État général & $\begin{array}{l}\text { Eramen des selles } \\
\text { Résultat }\end{array}$ \\
\hline $\begin{array}{l}24-7-1951 \\
25-7-1951 \\
26-7-1951\end{array}$ & $\begin{array}{c}40 \mathrm{cg} \\
- \\
-\end{array}$ & R.A.S. & $27-7-1951$ & $\begin{array}{l}\text { Aucune amé- } \\
\text { li or a tion } \\
\text { des signes } \\
\text { cliniques } \\
\text { Apparition } \\
\text { d'ascite. Ab- } \\
\text { domen dis- } \\
\text { tendu. }\end{array}$ & Négatif. \\
\hline $\begin{array}{r}30-7-1951 \\
31-7-1951 \\
1-8-1951\end{array}$ & $\begin{array}{c}35 \mathrm{cg} \\
- \\
-\end{array}$ & Ptyalisme & $6-8-1951$ & $\begin{array}{l}\text { Appétit } \\
\text { retrouvé }\end{array}$ & Négatif. \\
\hline $\begin{array}{r}30-7-1951 \\
31-7-1951 \\
1-8-1951\end{array}$ & $\begin{array}{c}40 \mathrm{cg} \\
- \\
-\end{array}$ & R.A.S. & $\begin{array}{c}6-8-1951 \\
13-10-1951\end{array}$ & $\begin{array}{l}\text { Bon état } \\
\text { général }\end{array}$ & Négatif. \\
\hline $\begin{array}{l}1-8-1951 \\
2-8-1951 \\
3-8-1951\end{array}$ & $\begin{array}{l}1 \mathrm{~g} \\
-\end{array}$ & R.A.S. & $\begin{array}{c}4-8-1951 \\
6-8-1951 \\
13-10-1951\end{array}$ & $\begin{array}{l}\text { Léger } \\
\text { appétit }\end{array}$ & $\begin{array}{c}\text { Négatif. } \\
- \\
-\end{array}$ \\
\hline $\begin{array}{l}1-8-1951 \\
2-8-1951 \\
3-8-1951\end{array}$ & $\begin{array}{c}40 \mathrm{cg} \\
- \\
-\end{array}$ & R.A.S. & $\begin{array}{l}4-8-1951 \\
6-8-1951\end{array}$ & $\begin{array}{l}\text { Bon état } \\
\text { Guérison }\end{array}$ & Négatif. \\
\hline $\begin{array}{l}2-8-1951 \\
3-8-1951 \\
4-8-1941\end{array}$ & $\begin{array}{c}45 \mathrm{cg} \\
- \\
-\end{array}$ & R.A.S. & $8-8-1951$ & $\begin{array}{c}\text { Légère } \\
\text { amélioration }\end{array}$ & Négatif. \\
\hline $\begin{array}{l}5-8-1951 \\
6-8-1951 \\
7-8-1951\end{array}$ & $\begin{array}{c}1,65 \mathrm{~g} \\
- \\
-\end{array}$ & R.A.S. & $\begin{array}{l}16-8-1951 \\
13-8-1951 \\
30-8-1951\end{array}$ & $\begin{array}{c}\text { Pas } \\
\text { d'amélioration }\end{array}$ & $\begin{array}{c}\text { Positif } \\
- \\
-\end{array}$ \\
\hline $\begin{array}{l}6-8-1951 \\
7-8-1951 \\
8-8-1951\end{array}$ & $\begin{array}{c}1,40 \mathrm{~g} \\
- \\
-\end{array}$ & R.A.S. & $\begin{array}{r}\cdot 14-8-1951 \\
21-8-1951 \\
30-8-1951\end{array}$ & $\begin{array}{c}\text { Légère } \\
\text { amélioration }\end{array}$ & $\begin{array}{c}\text { Négatif. } \\
- \\
-\end{array}$ \\
\hline $\begin{array}{l}7-8-1951 \\
8-8-1951 \\
9-8-1951\end{array}$ & $\frac{2 \mathrm{~g}}{-}$ & R.A.S. & $\begin{array}{r}18-8-1951 \\
28-8-1951 \\
5-9-1951\end{array}$ & $\begin{array}{c}\text { Légère } \\
\text { amelioration }\end{array}$ & $\frac{\text { Négatif. }}{\text { Positif }+}$ \\
\hline $\begin{array}{l}7-8-1951 \\
8-8-1951 \\
9-8-1951\end{array}$ & $\begin{array}{l}1 \mathrm{~g} \\
- \\
-\end{array}$ & R.A.S. & $\begin{array}{r}18-8-1951 \\
28-8-1951 \\
5-9-1951\end{array}$ & $\begin{array}{c}\text { Pas } \\
\text { d'amélioration }\end{array}$ & $\begin{array}{c}\text { Positif } \\
- \\
-\end{array}$ \\
\hline
\end{tabular}

